\title{
Analysis of Remote Islanding Detection Methods for Distributed Resources
}

\author{
A. Etxegarai, P. Eguía, I. Zamora \\ Department of Electrical Engineering - University of the Basque Country \\ Escuela Técnica Superior de Ingeniería de Bilbao \\ Alda. Urquijo s/n, 48013 Bilbao (Spain) \\ e_mail: agurtzane.etxegarai@ehu.es
}

\begin{abstract}
Penetration of distributed generation in the power grid is increasing. This situation leads to new possibilities but also new issues, such as anti-islanding protection. During a loss-of-mains or islanding situation, distributed generation (DG) units remain feeding a part of the electrical network without connection to the main power system. Currently, DG units have to be disconnected from the grid during islanding as soon as possible, due to potential risks for repair crews and components, poor power quality in the island and reclosing problems. A fast and reliable islanding detection method could avoid mentioned issues. Among the many existing methods, remote schemes could meet these requirements.
\end{abstract}

Hence, this paper presents the operating principles of remote islanding detection methods, as well as the current state of development of communication-based remote islanding detection methods to be applied in distributed power generation systems.

Key words: distributed generation, loss of mains protection, islanding detection, IEC 61850, synchrophasor, PMU.

\section{Introduction}

During an islanding situation a portion of the power system, which contains both load and distributed resources, remains energized, while it is isolated from the rest of the grid, forming an uncontrolled island. This operation can lead to hazardous situations. Therefore, it is important to detect unintended electric islands within a short time delay and to trip the islanded generators $[1,2]$.

Islanding detection techniques are usually divided into local detection methods, when the detection is based on the DG side, and remote detection methods, when the detection is based on the utility side. Remote antiislanding schemes allow disconnection decisions to be made by the utility company [3]. Most of the latter methods rely on external communication devices which link each feeder to the utility side.
Local detection methods, in turn, can be classified into passive and active techniques [4]:

- Passive methods rely on available local measurement to detect islanding situations. Actually, sudden islanding of a grid causes changes in some electrical parameters, such as frequency and voltage. The most used passive methods are therefore over/under voltage and frequency relays. Other techniques are based on harmonic distortion, voltage phase and rate of change of frequency measurements $[5,7,8]$.

- Active methods directly interact with the power system operation by introducing small perturbations. These small perturbations result in a significant change in system parameters when the distributed generator is islanded, whereas the change is negligible when it is connected to the grid. Some active methods are phase shift methods, impedance measurement method and reactive power export method $[6,7,8]$.

A proper evaluation of loss-of-mains detection methods should consider the following aspects [7, 9, 10], summarized in Table I:

- Dependability and security: they are related to the reliability of the island detection, which is normally assessed by non-detection zones (NDZ). NDZs are regions in a defined space in which the islanding detection scheme fails to detect islanding [1].

- Operating time: IEEE 1547 Std. defines maximum operation delays (2s), which must include islanding detection latency and tripping time. Operating time must be shorter than the recloser acting time.

- Impact on the grid: the application of some techniques may degrade the power quality, and impact on the transient response of the power system.

- Cost of the solution.

- Adaptability to grid characteristics: islanding detection depends on grid topology, generator technology and distributed generation penetration level. 
Table I. - Comparison of remote and local anti-islanding methods.

\begin{tabular}{|c|c|c|c|}
\hline & Remote methods & Local passive methods & Local active methods \\
\hline Dependability and security & $\begin{array}{c}\text { No NDZ } \\
\text { No nuisance trips }\end{array}$ & $\begin{array}{c}\text { Large NDZ } \\
\text { Possible nuisance trips }\end{array}$ & Few nuisance trips \\
\hline Operating time & Depends on the method & Short & Large \\
\hline Impact on grid & No impact & No impact & Possible impact \\
\hline Cost & High & Low & Low \\
\hline Adaptability to grid characteristics & Adequate adaptability & Adequate adaptability & $\begin{array}{c}\text { Depends on generators } \\
\text { technology and penetration } \\
\text { level }\end{array}$ \\
\hline
\end{tabular}

In general, it can be said that the performance of local detection methods is often unsatisfactory, because of large NDZ for passive methods or the impact on the grid and dependence on grid characteristics for active methods. However, remote islanding detection techniques overcome these issues, because they are independent of local power balance, do not inject perturbations on the grid and can be adapted for different grid scenarios, as shown in Table I. Unfortunately, two critical aspects must be also highlighted: operating times and high costs.

This paper presents remote islanding detection operating principles and recent communication-based islanding detection systems. These methods are evaluated taking into account the most critical aspects.

\section{Remote Islanding Detection Operation Principles}

Remote islanding detection algorithms are located at the utility level. Although these techniques may have better reliability than local techniques, they are usually expensive to implement and islanded distributed generator tripping times are variable. Following, some remote islanding detection operation principles are outlined as the most representative.

\section{A. Impedance insertion}

This method inserts a low-value impedance, usually a capacitor bank, when the utility breaker opens creating an electric island. As a result, the power balance between generation and load is modified, because reactive power is out of balance [6]. Also, voltage and frequency change in the distribution line. The frequency deviation is detected by the utility side frequency relay. Fig. 1 shows a scheme of the operating principle:

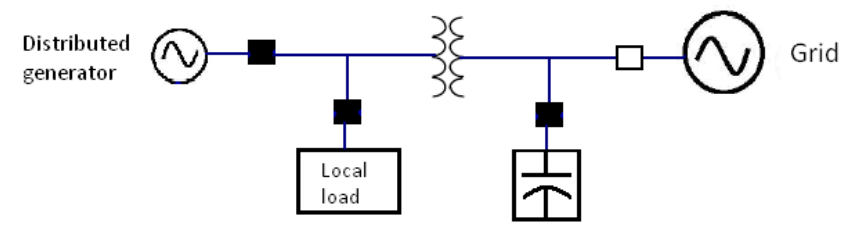

Fig. 1. Scheme of the impedance insertion method.
Although this method has been proven to be highly effective in islanding detection [11], it has also important weaknesses, such as:

- Cost of the capacitor. Nevertheless, the same capacitor bank could be used for other purposes, such as reactive power (voltage) support.

- Low response time, partly due to the switching time of the capacitor, but also to the necessary delay in switching to allow the frequency deviation to be detected. Detection times can be above certain standards, such as [12]. Though, the islanding detection time decreases as the capacitance increases [11], obtaining standard complying delays with relatively small capacities, about $20 \%$ of the generation capacity [13]. Therefore, the impedance value should be sized according to the minimum variation of frequency that can be detected.

\section{B. Power Line Carrier Communications (PLCC)}

This method uses the power line as signal carrier. As distributed generators are located in the distribution network, it is usually called Distribution Line Carrier (DLC). A signal generator located at the distribution substation is continuously sending broadcast signals to all distributed generators, which are equipped with signal detectors. As soon as islanding happens, the broadcast signal is not anymore detected by the DG side signal receivers and therefore, all $\mathrm{DG}$ units are tripped. If distance between substation and DG unit exceeds $15 \mathrm{~km}$, the signal would be attenuated, and therefore, repeaters would be necessary $[14,16]$. Fig. 2 shows a scheme of this operating principle:

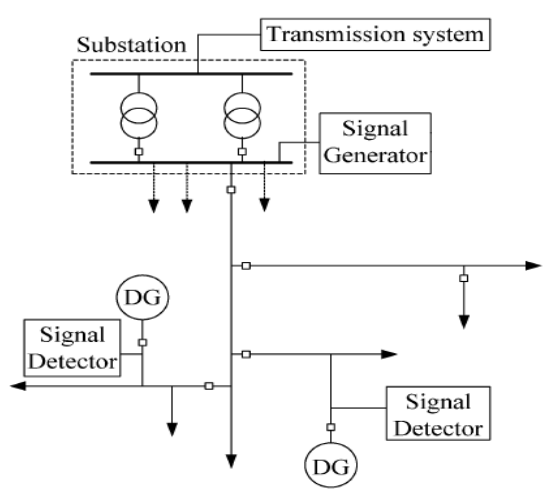

Fig. 2. PLC-based islanding detection scheme [15]. 
This detection scheme has a good speed of response [15]. As only two cycles are needed to carry one pulse, the method could detect islanding in those two cycles. Nevertheless, to avoid excessive nuisance trips, longer pulses are usually used, and missing four consecutive pulses is considered an islanded condition. The major disadvantages of this scheme are:

- The implementation cost of the system, in the utility side but also in the distributed generation side. Though, no additional wires are needed, as in the case of other communication-based methods, because power line is used.

- This method could have a small NDZ when using a subharmonic signal [14], which is not frequent.

\section{Transfer trip schemes}

Transfer trip detection method requires all circuit breakers which island the DG to be monitored and linked to a central control unit. When a disconnection is detected at the substation, the central algorithm determines which areas are islanded, and sends the appropriate signal to generators, to either remain in operation, or to trip. Supervisory Control and Data Adquisition (SCADA) systems can be used for this purpose. Fig. 3 shows a scheme of this operating principle.

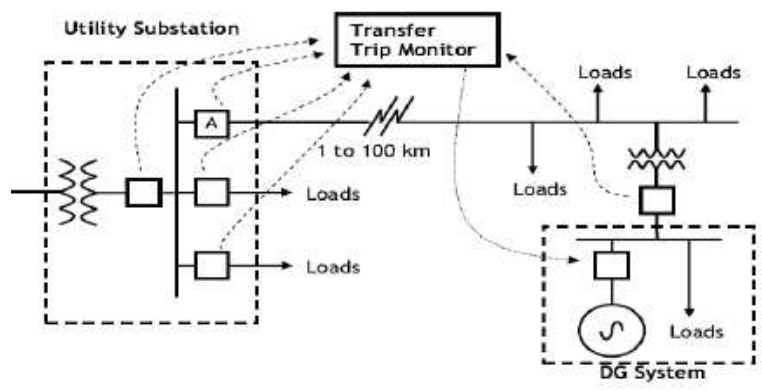

Fig. 3. Transfer trip scheme.

Transfer trip schemes require obviously extensive communication support. Traditionally, radio communication or leased telephone lines have been the most common media. Though, nowadays new communication means have been introduced. For instance, Internet broadband, wireless communication, optic fiber Ethernet and satellite communication have been proposed, together with new communication protocols such as IEC61850. These new approaches will be further discussed in section 3 .

Like the previous detection methods, cost is a serious drawback for transfer trip schemes, as well as a design complication. These disadvantages increase as the grid becomes more complex, and redesign might have to be considered.

\section{Comparative analysis of remote islanding detection operation principles}

High costs and long operating times have been previously highlighted as the main drawbacks of remote islanding detection techniques. Therefore, the aforementioned remote detection operation principles are evaluated considering these aspects in Table II.

Table II. - Comparison of remote anti-islanding protection methods

\begin{tabular}{|l|l|l|l|}
\hline & $\begin{array}{l}\text { A. Impedance } \\
\text { insertion }\end{array}$ & B. PLCC & C. Transfer trip \\
\hline $\begin{array}{l}\text { Cost } \\
\text { includes }\end{array}$ & - Capacitor & $\begin{array}{l}- \text { Transmitter } \\
- \text { Receiver } \\
- \text { Coupling } \\
\text { device } \\
- \text { Repeater }\end{array}$ & $\begin{array}{l}\text { - Dedicated } \\
\text { communication } \\
\text { lines } \\
\text { - SCADA }\end{array}$ \\
\hline $\begin{array}{l}\text { Operating } \\
\text { time }\end{array}$ & $1 \mathrm{~s}<\mathrm{t}<2 \mathrm{~s}$ & $277 \mathrm{~ms}$ & $\begin{array}{l}\text { Depends on the } \\
\text { communication } \\
\text { link }\end{array}$ \\
\hline
\end{tabular}

The cost related to the implementation of remote island detection schemes are inherently high, but could be partly shared considering that the capacitors required used in method A could also be used for voltage support. As for communication-based $\mathrm{B}$ and $\mathrm{C}$ methods, communication links are nowadays widely also used for power system automation.

In relation with operating times, capacitor connection methods have important latencies. Therefore, they should be used as back-up protection. PLCC systems have low operating times, and in the case of transfer trip schemes, these delays depend strongly on the communication link.

\section{Smart Islanding Detection Methods}

The distribution grid is evolving to a smarter grid, where communications between intelligent electronic devices (IEDs) play an important role. The IEDs are microprocessor-based controllers of the power grid equipment, which can receive and transmit data, as well as send commands. This way, they contribute to power grid automation [23]. Some new communication-based islanding detection systems have been proposed following this tendency. The new approaches are flexible and easily adaptable to grid configuration changes, as well as future grid requirements. Furthermore, compared to traditional remote antiislanding protection, they might be economically feasible.

Last coming remote islanding detection techniques have made important breakthroughs regarding the following aspects:

- Operating principles: island detection based on synchrophasor $[17,18]$, new inter-tripping schemes [20] and detection based on distributed control [21].

- Communication link: internet-based scheme [22].

- Protocol: some new remote islanding detection techniques $[20,21]$ are based on the protocol IEC 61850 , a new communication standard for substation automation, which ensures interoperability between IEDs and configuration adaptation. IEC 61850 can be 
applied in vertical and horizontal communication between IEDs. GOOSE (Generic Object Oriented Substation Event) type messages are intended for transmitting point-to-point events between IEDs at substation level, instead of using hardwired systems, whose operation is quite slow.

\section{A. Operating principles}

Following are presented some new islanding detection methods which are based on innovative operating principles.

\section{A.1. PMU-based islanding detection method}

In this method, the loss of the grid is detected by the algorithm of a central synchrophasor vector processor, which uses synchrophasor data from two relays, located at the grid side and at the distributed generator side (Fig. 4).

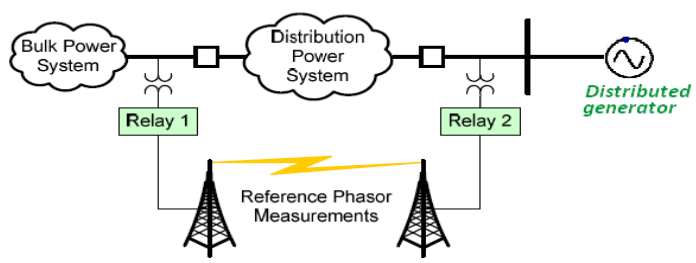

Fig. 4. PMU-based islanding detection method [17].

Two techniques concerning synchrophasors for islanding detection are being used $[17,18]$ :

- Angle difference method: in this method, difference between the local and remote synchrophasor angle value are compared against an angle threshold. If the angle difference is greater than the threshold, the logic detects an islanding condition and sends a tripping command to the circuit breaker.

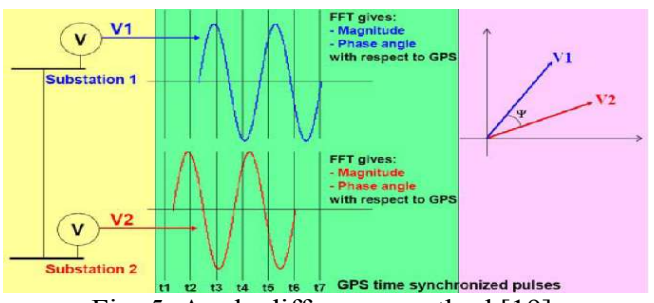

Fig. 5. Angle difference method [19].

- Slip-acceleration method: this method monitors the rate-of-change of phase. This parameter is defined as the slip and the rate-of-change of the slip as acceleration. Combining slip and acceleration creates the islanding detection characteristic shown in Fig. 6.

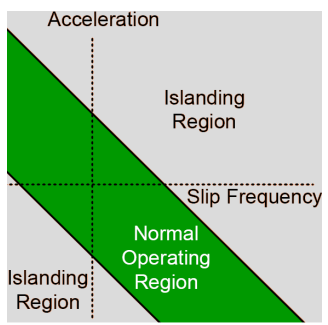

Fig. 6. Slip-acceleration islanding detection characteristic [17].
If the operating point is in the grey islanding region of Fig. 6, island situation is readily detected. In general, for PMU-based islanding detection, the response time includes the latencies shown in Fig. 7:

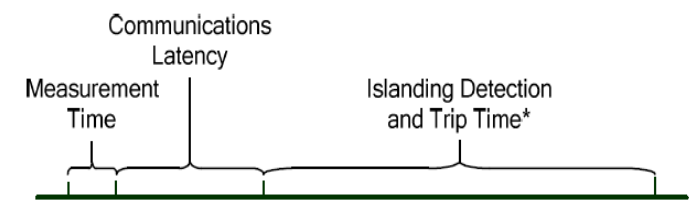

Fig. 7. Total time to trip DG in case of islanding [17].

Communications latency is related to the coordination of the reference measurement with the multiple generator locations. Response delays are generally smaller in local islanding detection schemes. Nevertheless, PMU-based detection is faster than local methods in some particular cases when generation matches load.

\section{A.2. Centralized Islanding Detection method (CID) [20]}

It is a new inter-tripping scheme without a predetermined logic, as conventional schemes. Thus, it is very flexible, allows changes in network topology and is easily extendable. The islanding detection algorithm is installed in a central controller connected to all IEDs in the network (Fig. 8). This method includes not only a innovative operating principle, but also a recent protocol. Thus, the data modeling and communication protocol is based on IEC 61850 standard with an Ethernet link. The central controller monitors the status of the circuit breakers and sends tripping commands to the distributed generators in case of islanding, using GOOSE messages. The detection scheme can be extended to multiple generators and applied to any network.

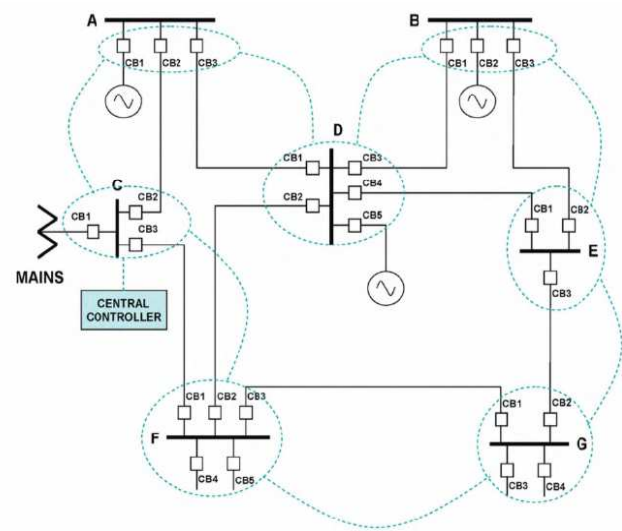

Fig. 8. Centralized Islanding detection method [20].

This method has been proven to be robust, reliable and operating time delay is standard compliant [12].

\section{A.3. Distributed controlled islanding detection [21]}

Islanding protection can be developed in a fast, reliable and selective way through the communication link of a modern line differential protection. The differential relay's communication channels are used to apply a 
transfer-trip scheme based detection. A combination of vertical inter-substation BST (Binary Signal Transfer) signaling with horizontal IEC 61850 GOOSE messaging at the substation level is proposed, in order to enhance the operation and the reliability of the anti-islanding protection (Fig. 9).

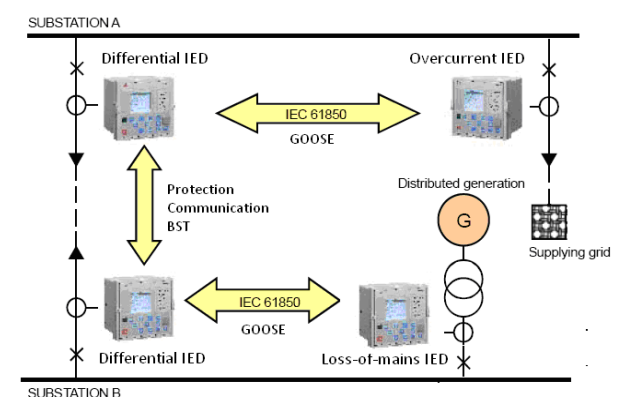

Fig. 9. Islanding detection included in the line differential protection [21].

The total transfer times must include the physical binary input activation delay, GOOSE message transfer delay, BST signaling delay and the physical output contact delay. Altogether, the operating speed of this scheme can be much faster than traditional detection methods.

\section{B. Communication link}

When considering large DGs, a leased-circuit communications channel is cost justified. However, for small DGs the size and potential number of units makes a conventional leased-circuit SCADA implementation unattractive from a cost perspective. Latest experiences show interesting results using domestic broadband Internet connections as communication support for realtime power system protection [18,22]. A Virtual Private Network (VPN) is implemented between stations (Fig. 10). Protection signals such as circuit-breaker status are broadcasted through Internet. This broadcast signals' data are encapsulated in a 'ping' message data payload.

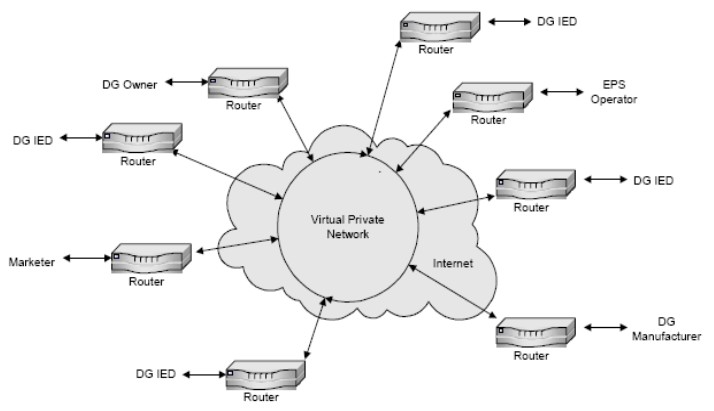

Fig. 10. Internet-based islanding detection: VPN [22].

Internet-based islanding detection is performed with the following operation principles, already mentioned:

- Reference frequency and voltage phasor measurements are broadcasted over Internet and compared to the measurements in the DG side, or

- Transfer trip scheme: a server system broadcasts utility side circuit breakers' status information to distributed generators' side relays, via Internet. When an island is detected, distributed generators are tripped.

\section{Comparative analysis of smart islanding detection methods}

Deployment cost as a critical drawback of remote islanding detection methods seems to be nowadays overshadowed by their technical good performance. Operating times must be therefore carefully analyzed, including communication latencies, as smart techniques are all communication-based schemes, as well as island detection algorithm processing delays.

Internet as a new communication link for communication-based islanding detection schemes offers real-time communication but with a degree of unreliability caused by the fundamental "best-effort" protocol, especially in wireless networks. Thus, although trip times might be well under grid codes requirements, with only $160 \mathrm{~ms}$ communication latency time for an ADSL link [22], back-up protection might be necessary to ensure reliable operation. Communication-due delay is, in turn, $100 \mathrm{~ms}$ in CID system, being Ethernet the communication link [20]. GOOSE message-based systems have also low communication delays [21].

Island detection algorithm processing delays must also taken into account. PMU-based systems need 1.25 seconds in total to trip an islanded DG unit. In turn, CID system algorithm needs only $75 \mathrm{~ms}$ to detect islanding. The aforementioned operating times, communication delay and detection time included, are showed in Table III.

Table III. - Comparison of remote islanding detection methods.

\begin{tabular}{|l|l|l|l|l|}
\hline & A.1. & A.2. & A.3. & B \\
\hline $\begin{array}{l}\text { Operating } \\
\text { times }\end{array}$ & $\begin{array}{l}1.15- \\
1.7 \mathrm{~s}\end{array}$ & $\begin{array}{l}185- \\
275 \mathrm{~ms}\end{array}$ & $30 \mathrm{~ms}$ & $160 \mathrm{~ms}\left(^{*}\right)$ \\
\hline
\end{tabular}

(*) Only communication latency is considered.

Although some methods are especially fast, all of them are standard compliant [12], being the operating time less than 2 seconds. It can generally be stated that remote smart islanding detection systems are faster than traditional remote schemes.

\section{Conclusions}

This paper has presented an analysis of characteristics and operation principles of remote islanding detection methods. It especially describes the most recent projects, which are either innovative for their operating principle, communication link or protocol. These new islanding detection architectures will fit in the future smart grid, providing quality power supply and additional services to customers.

The smart anti-islanding protection schemes, as well as traditional remote schemes, have the following advantages against local methods:

- Their performance is independent of the type of distributed generators involved. The operation is also correct in case of multiple DG units. 
- They are highly reliable and robust. They do not have a substantial NDZ.

- Their power quality impact is not perceptible.

Besides, they overcome some of the traditional weaknesses of remote methods:

- The smart solutions can be economically feasible, because they can use existing communication links and the detection algorithm can be integrated in other protection relays, sharing the total costs.

- They are flexible and can be easily adapted to network topology changes or system extension.

Detection time is, still, an important issue, as it strongly depends on communication latencies and therefore, on the communication link. Combining local and remote anti-islanding protection could be a solution for overcoming this short-coming. This way, under normal communication conditions, a fast islanding detection is possible with the aforementioned smart remote methods. If the communication link is down or communication latencies are high, anti-islanding protection can rely on local schemes, acting as back-up protection.

\section{Acknoledgement}

The work presented in this paper has been supported by the Basque Government (Ref. IT532-10).

\section{References}

[1] M. Ropp, M. Begovic, A. Rohatgi, "Prevention of Islanding in Grid-Connected Photovoltaic Systems", Progress in Photovoltaics vol. 7 (1999), p. 39-59.

[2] N. Strath, "Islanding detection in power systems," Ph.D. dissertation, Lund University, 2005.

[3] W. Xu, K. Mauch, and S. Martel, "An assessment of distributed generation islanding detection methods and issues for Canada," CANMET Energy Technology Centre - Varennes, Natural Resources Canada, QC-Canada, Tech. Rep. CETC-Varennes 2004-074 (TR), 2004].

[4] W. Bower and M. Ropp, "Evaluation of Islanding Detection Methods for Photovoltaic Utility-Interactive Power Systems", International Energy Agency Task V Working Group report IEA-PVPS T5-09, March 2002.

[5] F. De Mango et al, "Overview of Anti-Islanding Algorithms for PV Systems. Part I: Passive Methods", EPE-PEMC 2006, Portoroz (Slovenia).

[6] F. De Mango et al, "Overview of Anti-Islanding Algorithms for PV Systems. Part I: Passive Methods", EPE-PEMC 2006, Portoroz (Slovenia).

[7] T. Funabashi, K. Koyanagi, and R. Yokoyama, "A review of islanding detection methods for distributed resources," in Proc. IEEE Power Tech Conf., vol. 2, Bologna, June 2004.

[8] P. Mahat, Z. Chen, and B. Bak-Jensen, "Review of islanding detection methods for distributed generation," in Proc. 3rd Int. Conf. Electric Utility Deregulation and Restructuring and Power Technologies, Apr. 6-9, 2008, pp. 2743-2748.
[9] O. Samuelsson, N. Strath, "Islanding detection and connection requirements," Power Engineering Society General Meeting, 2007. IEEE, pages: 1 - 6, 24-28 June 2007.

[10] Z. Ye, A. Kolwalkar, Y. Zhang, P. Du, and R. Walling, "Evaluation of anti-islanding schemes based on nondetection zone concepts," IEEE Trans. Power Electronics, Vol. 19, no.5, pp. 1171-1176, Sept. 2004.

[11] A. Kitamura et al, "Islanding Phenomenon Elimination Study at Rokko Test Center", Proceedings of the 1st World Conference on Photovoltaic Energy Conversion, 1994, pt. 1, pp. 759-762.

[12] IEEE Standard for interconnecting distributed resources with electric power systems, IEEE Standard 1547-2003, June 2003.

[13] H. Kobayashi, K. Takigawa, E. Hashimoto, E. (1991). Method for Preventing Islanding Phenomenon on Utility Grid with a Number of Small Scale PV Systems, Proc. of the 21st IEEE Photovoltaic Specialists Conference, pp. 695-700, U.S.A.

[14] R. Benato, R. Caldon, F. Cesena, "Carrier signal-based protection to prevent dispersed generation on $\mathrm{MV}$ systems", CIRED 2003.

[15] W. Xu et al., "A power line signaling based technique for anti-islanding protection of distributed generators", IEEE Transactions on Power Delivery, vol. 22, no. 3, July 2007.

[16] O. Abarrategui, I. Zamora, D.M. Larruskain, M. Gomez "DLC Communications for Islanding Detection in Systems with DG” XCLEE Madeira, Portugal, July 2007.

[17] E. O. Schweitzer et al., "Synchrophasor-Based Power System Protection and Control Applications", Modern Electric Power Systems 2010.

[18] R.J. Best et al. , "Synchrophasor Broadcast Over Internet Protocol for Distributed Generator Synchronization", IEEE Transanction on Power Delivery 2010, Vol. 25, no.4, pp. 2835-2841, Oct. 2010.

[19] M. Patel, "PJM SynchroPhasor Technology Deployment Project”, EEI Conference 2010.

[20] F. Coffele, P. Moore, et al. "Centralised Loss of Mains protection using IEC-61850", 10th IET International Conference on Developments in Power System Protection (DPSP 2010).

[21] O. Rintamaki, K. Kauhaniemi, "Applying modern communication technology to loss-of-mains protection", CIRED 2009.

[22] D. Laverti, D.J. Morrow, T. Littler, "Internet based loss-ofmains detection for distributed generation", UPEC 2007.

[23] A. Timbus et al, "Islanding detection in smart grids", ECCE 2010, pp.3631-3637, Sept. 2010. 